\title{
A Research on Different Filtering Techniques and Neural Networks Methods for Denoising Speech Signals
}

\author{
G.K Rajini, V.Harikrishnan, Jasmin Pemeena Priyadarisini M, S.Balaji
}

\begin{abstract}
This paper intends to provide the best suited noise removal technique for de-noising and retrieving clean speech from a noisy speech signal. The aim is to use different de-noising techniques and compare their performance and arrive at a conclusion regarding which one of them is best suited for enhancing voice signals. The analysis is done by evaluating the performance of different denoising techniques for different types of speech samples. This evaluation is done by adding random noise to speech signal then applying denoising techniques to get denoised speech signal. A parallelism is drawn between original signal and denoised signal through evaluation parameters such as SNR and PSNR. The denoising methods are broadly classified as 'The Filtering Methods' and 'The Neural Network Methods'. Under filtering methods four different denoising methods have been used. The four different denoising methods are - Adaptive Filter based on LMS Algorithm, Weiner Filter, Chebyshev Filter and Kalman Filter. Under neural network methods we use three different denoising methods 'ADALINE' and two deep learning methods with 'Fully Connected' and 'Fully Convolutional' neural networks. The performance estimation is done based on variation of evaluation parameters (SNR and PSNR values) for different denoising techniques.
\end{abstract}

Keywords - Speech denoising techniques, SNR and PSNR evaluation, Filtering techniques, Neural Networks techniques

\section{INTRODUCTION}

The fundamental senses are hearing, sight, smell, taste and touch. These senses perceive the information from the environment and human brain processes this information to create a precise response. The complex communication between humans and their environment is aided by these senses. Sound acts as an information provider to these senses. The information has to be noise free for us to better understand the external environment. Noise can be described as any unwanted information which hinders the ability of the human body to process the valuable sensory information. Hence a noise free sound becomes essential for proper interaction of humans with their external world. The primary focus is on speech signals which are information providers in various communication systems. During the transfer of signals, distortion by some unwanted signals causes loss of useful data and information stored in the signals. There are many real time noise signals such as the noise of a train, traffic jam, washing machine and turbines

Revised Manuscript Received on July 18, 2019.

G.K Rajini, School of Electrical Engineering,Vellore Institute of Technology, Vellore, Tamilnadu, India

V.Harikrishnan, School of Electrical Engineering,Vellore Institute of Technology, Vellore, Tamilnadu, India

Jasmin Pemeena Priyadarisini $\mathbf{M}$, School of Electronics Engineering, Vellore Institute of Technology, Vellore, Tamilnadu, India

S.Balaji, School of Electrical Engineering,Vellore Institute of Technology, Vellore, Tamilnadu, India etc. which have to be reduced to retrieve the wanted information.

Speech signals are non-stationary, one dimensional signals produced by employing the speaker's lips, tongue, cheeks and nose. The frequency of speech signals ranges from $85 \mathrm{~Hz}$ to $255 \mathrm{~Hz}$. Typical male voices range lie in the lower frequency range between 85-180 Hertz whereas the female voices fall in the higher range of $165-255 \mathrm{~Hz}$. Babies have even higher ranges of frequency reaching up to $1000 \mathrm{~Hz}$ in a few cases. The sound is created by changing the air pressure with time. [2][3] This fluctuation in air pressure is captured in terms of voltages and currents to form electrical signals by microphones. When the voice signal is thus captured and communicated, it is often riddled with noise.

In the paper, the noise is added to a clean audio signal and then it is passed through the filter to obtain the denoised audio. The parameters being used to gauge the performance of the filters are PSNR and SNR. The motive is to obtain the best SNR and PSNR values for different types of sample speech signals and to provide the best suitable noise reduction method for a particular sample signal. There are variety of human spoken languages, each with different sound characteristics such as pitch, separation between vocal folds, tones etc. hence the speech signals contains samples of different languages in both male and female voice. The analysis of each noise cancellation methods, to put forward both its positive and negative aspects along with its compatibility with sample signals is the main focus of this paper. The noise cancellation techniques have various real time applications, but often the implementation of these methods is challenging. Efforts have been made in this aspect by introducing artificial neural networks to provide noise free speech signals and working with noises we hear on a daily basis.

$S N R=20 \times \log \frac{r m s \text { SIGNAL }}{r m s \text { NOISE }}$

$P S N R=20 \times \log \frac{M A X}{\sqrt{ } M S E}$

There are many methods available for noise cancellation in speech signal. This paper has a motive to provide a brief overview and working of few signal processing and neural network de-noising techniques, to bring out the characteristics and functionality of these techniques. This is done by testing the techniques with hundreds of sample signals and to analyse about the suitability of these methods for different types of samples.

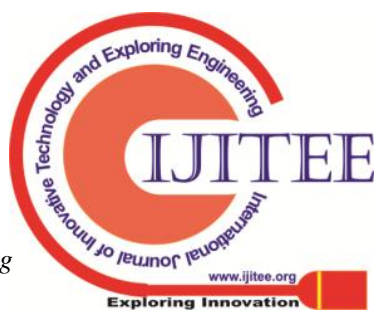




\section{THE FILTERING METHOD}

There are different filters used for de-noising speech signals. The filters that have been used are - Adaptive Filter using LMS (least mean square) algorithm, Weiner Filter, Chebyshev Filter and Kalman Filter.

\section{A. Adaptive Filter using LMS Algorithm}

Adaptive filter is like any other linear filter except that its transfer function is variable. It can be changed by adjusting some parameters. An error signal is generated by comparing the input signal and training signal and this is used to adjust the parameters. Thus, adaptive filters regulate themselves in accordance with the incoming signal. Adaptive filters have an algorithm to optimize the parameter's values. The LMS (Least mean square) algorithm employs the stochastic gradient descent technique. This means that the filter coefficients are only affected by the current value of error. As its name suggests the filter coefficients are directly related to the least mean square of the difference between the signal we desire and the original signal. The primary principle behind this approach is to keep updating the filter weights until they reach their optimum value. Initially, very small values are assigned to the weights and they are updated according to the MSE (Mean Square Error) gradient. The filter is adjusted to minimize the output power and the output noise power is also minimized. Hence by minimizing the total output power the filter maximizes the output signal-to-noise ratio.

While selecting the step size in the LMS algorithm one needs to be careful because if the step size is large the dependence of the weights on the gradient increases, so any minor change in the gradient would lead to oscillation of the weights. However, if the step size is too small the weights will take a very long duration of time to reach the ideal weight values.

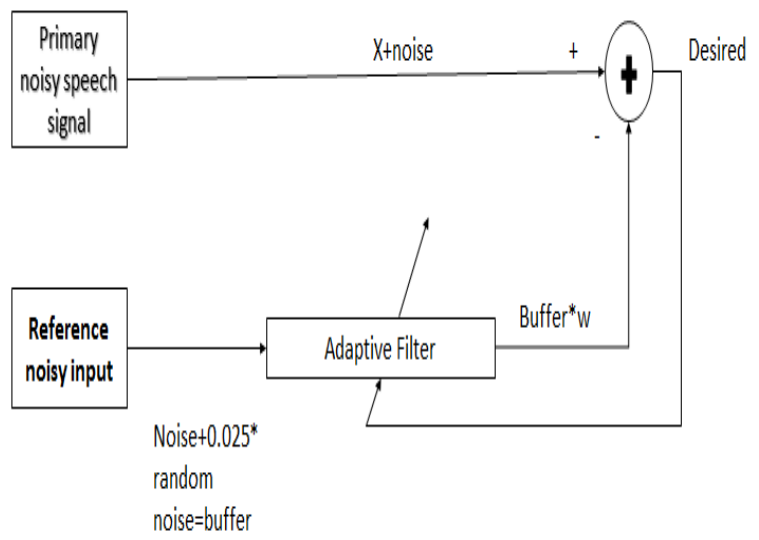

Fig. 1 Adaptive filter using LMS algorithm

In the Adaptive Filter, the step size can be varied. When the step size increases, computation time decreases, but error increases. On the other hand, when step size is reduced, computation time increases, but error decreases. This is illustrated in the table below.
Table. 1 Variation of MSE with Variation in Step Size

\begin{tabular}{|c|c|}
\hline STEP SIZE & MEAN SQUARE ERROR \\
\hline 0.01 & $5.43 \times 10^{-5}$ \\
\hline 0.06 & $1.88 \times 10^{-4}$ \\
\hline 0.1 & $3.83 \times 10^{-4}$ \\
\hline 0.5 & 0.0029 \\
\hline 1 & 0.0046 \\
\hline 5 & 0.0062 \\
\hline
\end{tabular}

The results of Adaptive Filter using LMS algorithm with graphs of the original, noisy, reference input denoised signals and the MSE plot have been illustrated below.
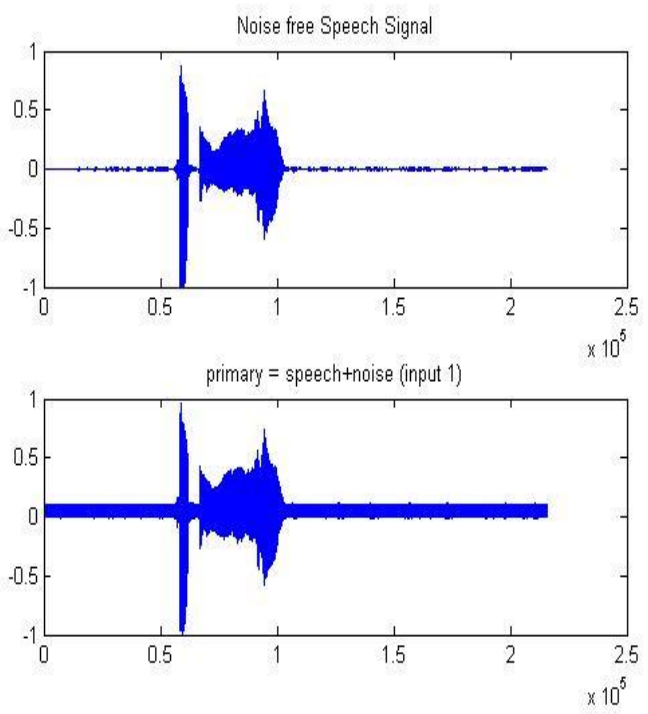

Fig. 2 Original and Noisy Speeches

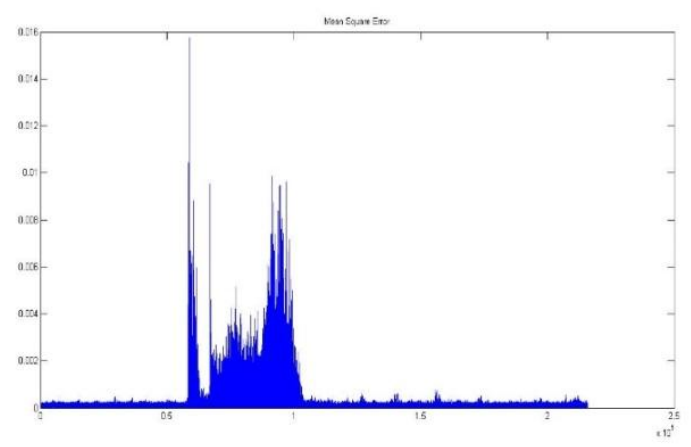

Fig. 3 Mean Square Error 


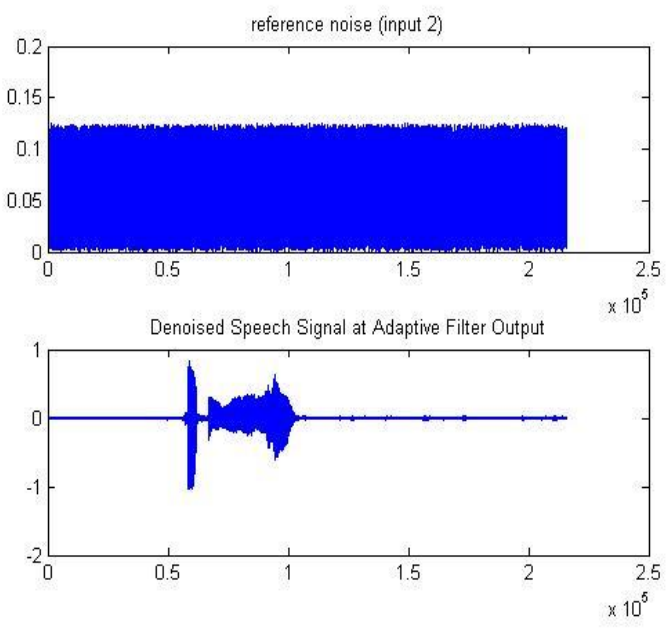

Fig. 4 Denoised Speech Signal

The adaptive filter using LMS algorithm cancels the noise from a noisy speech signal. The major advantage of this method include its ability to adapt to the noise, its tendency to provide output signals with low noise and to be able to do this without distorting the signal. Being an adaptive filter it can eliminate the unpredicted noise even when it has nonstationary properties.

\section{B. Weiner Filter}

Among the numerous techniques developed for noise cancellation one of the most fundamental is the Weiner filter. This filter reduces noise by drawing a comparison between the received signal and the estimate of the noiseless signal that we desire. However, in this filter the input is assumed to be stationary. For its implementation it is eminent that the user is aware of spectral properties (power functions etc.) of both the noise and the original audio.[12] The process works based on minimizing the mean square error between the new signal and the original signal. It is widely known that though the Weiner filter reduces the noise it comes at the cost of speech distortion. Therefore, it is only advised to use this filter when the noise cancellationspeech distortion trade-off is acceptable.

In this method Weiner filter is using the TSNR (Two Step Noise Reduction) Method. The signal observed is the combination of the clean audio and the noise. This speech is broken down into tiny frames. These frames are then overlapped with the successive frames. Each of them is smoothed using a Hamming window. Then using Fast Fourier Transform, the signal is converted from the time domain to the frequency domain. Phase and Magnitude are extracted from the FFT so obtained. The required modification to their value is calculated using the TSNR method. Once new phase and magnitude are obtained, their IFFT (Inverse Fast Fourier Transform) is calculated and the frames are overlapped and added to produce the denoised speech signal. [13]

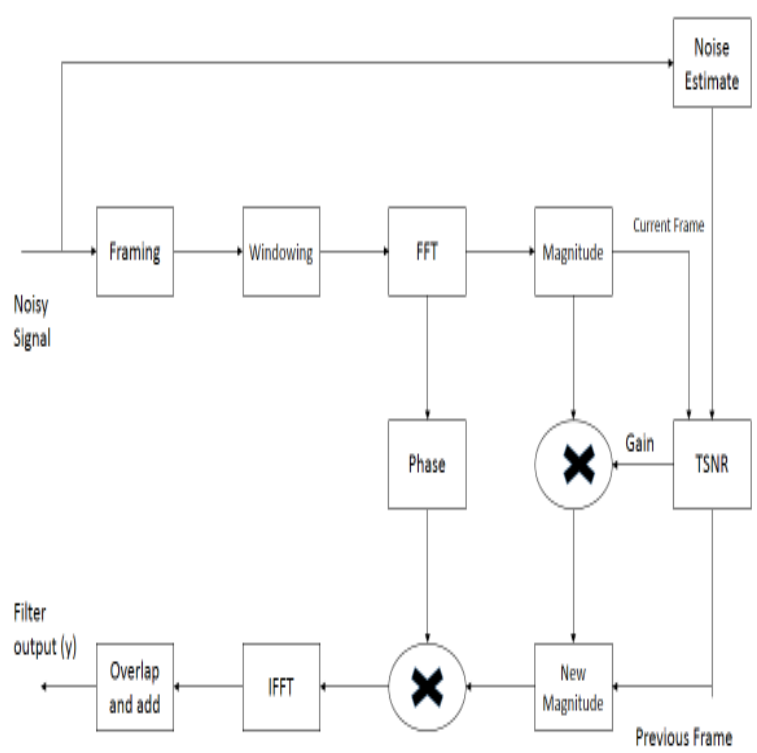

Fig. 5 Weiner filter using TSNR algorithm

The results of Weiner Filter with graphs of the original, noisy, denoised signals and the MSE plot have been illustrated below.

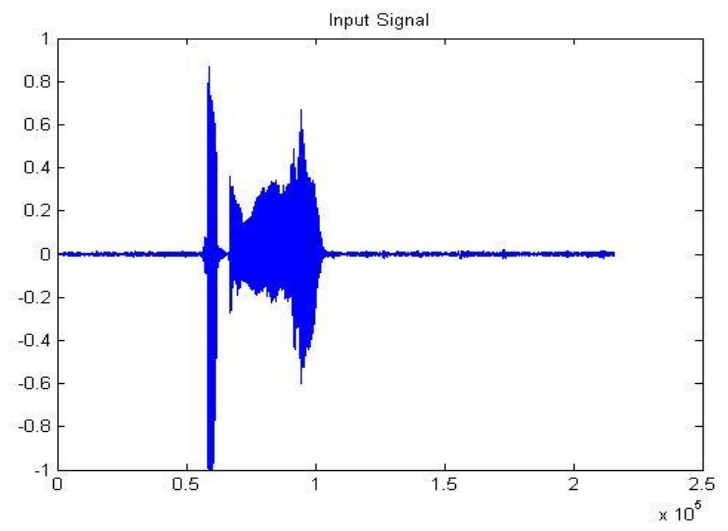

Fig. 6 Input Audio Signal

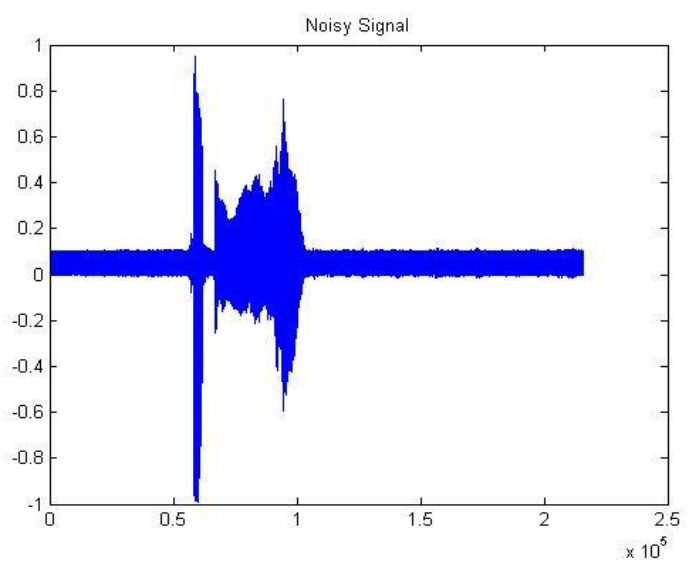

Fig. 7 Noisy Audio 


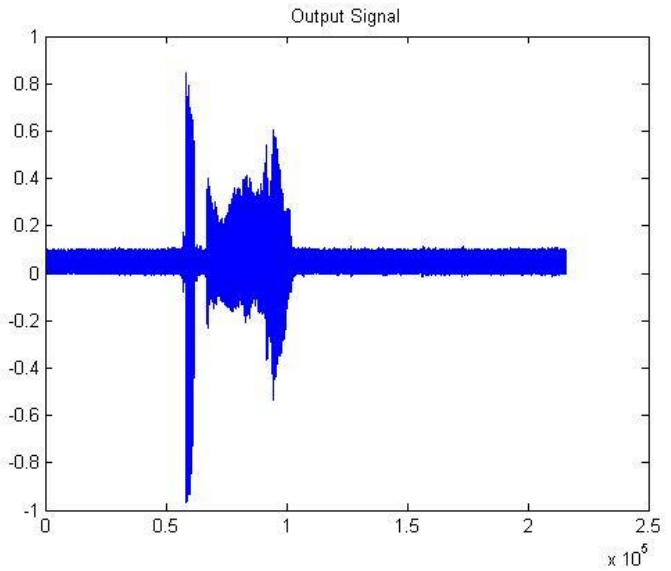

Fig. 8 Denoised Signal

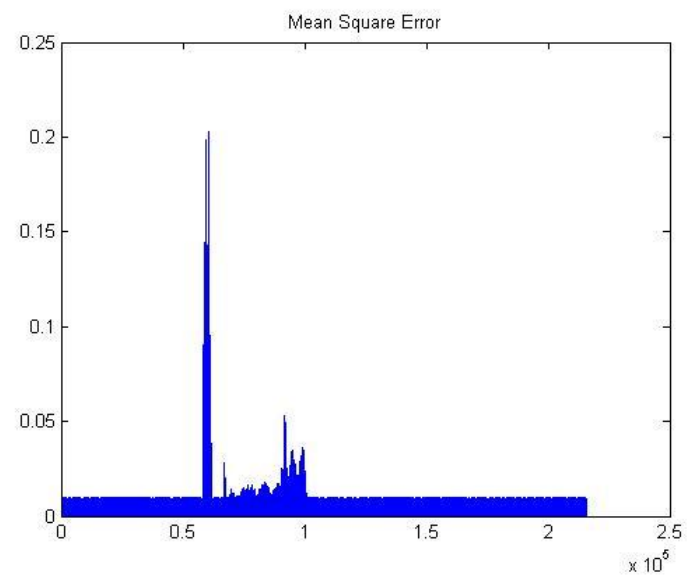

Fig. 9 Mean Square Error

The Weiner filter is the most fundamental technique used for noise cancellation in speech signals. This method causes speech degradation which reduces SNR and PSNR values. Hence, this method cannot be applicable for real time applications. However, there are some measures which performance of the filter by relating the amount of speech distortion with noise reduction factors.[12] The error is also relatively high as compared to other signals. Hence, it is not recommended to use this filter for noise cancellation in speech signals.

\section{Chebyshev Filter}

Chebyshev filters are very attractive as they are able to allow a particular band of frequency pass while blocking all others. Another advantage with these filters is that the time taken to process the signal is very less. This is because the Chebyshev filter's transition between its passband and stopband is extremely quick. However, these filters introduce ripples. [8][9]

The original audio file, which is in the .wav format is inputted into MATLAB. A random noise signal is added to the original audio. The input signal's frequency is set as the sampling frequency. The other parameters such as the Nyquist Frequency Fn, the passband frequency Wp, the stopband frequency Ws, the passband ripple Rp and the stopband ripple Rs are duly assigned. (In this particular paper their values are as $\mathrm{Fn}=($ Sampling Frequency $) / 2, \mathrm{Wp}$ $=1000 / \mathrm{Fn} ; \mathrm{Ws}=1010 / \mathrm{Fn} ; \mathrm{Rp}=1 ; \mathrm{Rs}=15)$. The filter is then designed using the inbuilt command in MATLAB. To double the order of the filter, zero phase digital filtering is used. Finally, the noisy audio is passed through the filter and a de-noised signal is obtained.
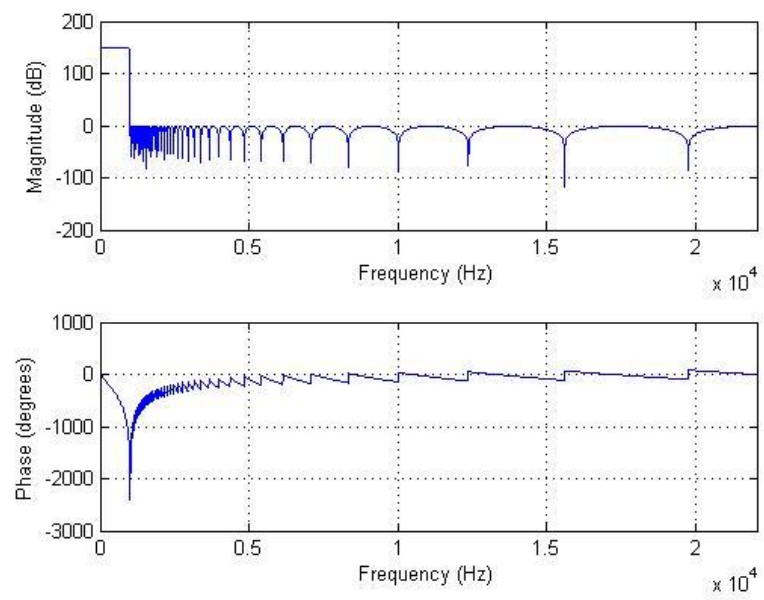

Fig. 10 Filter Plot

The results of Chebyshev Filter with graphs of the original, noisy, denoised signals and the MSE plot have been illustrated below.

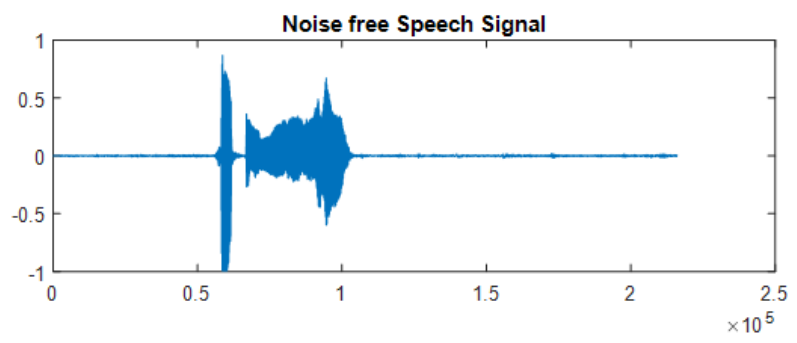

Fig.11 Noise Free Speech Signal

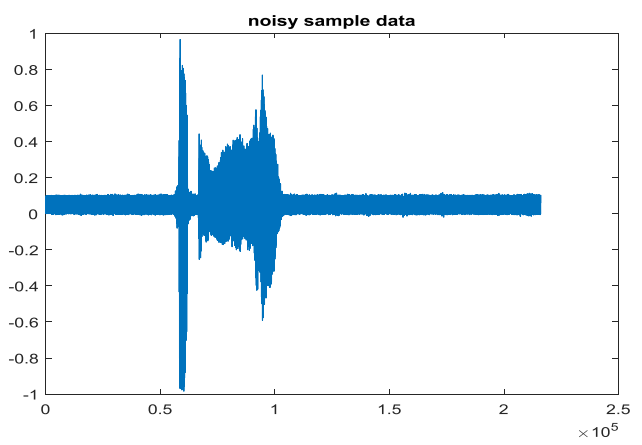

Fig.12 Noisy Sample Data

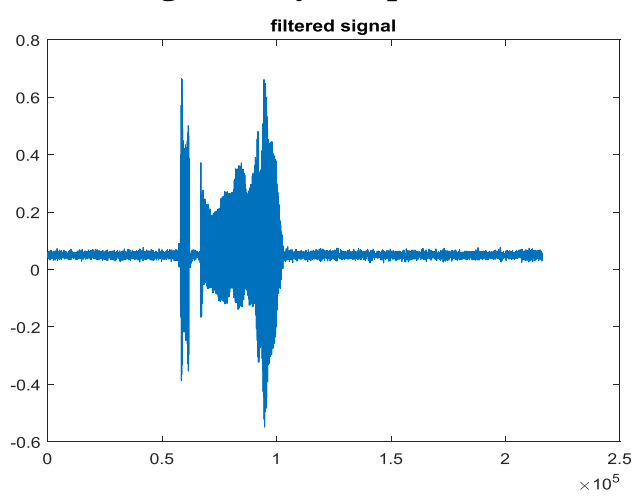

Fig .13 Filtered Signal 


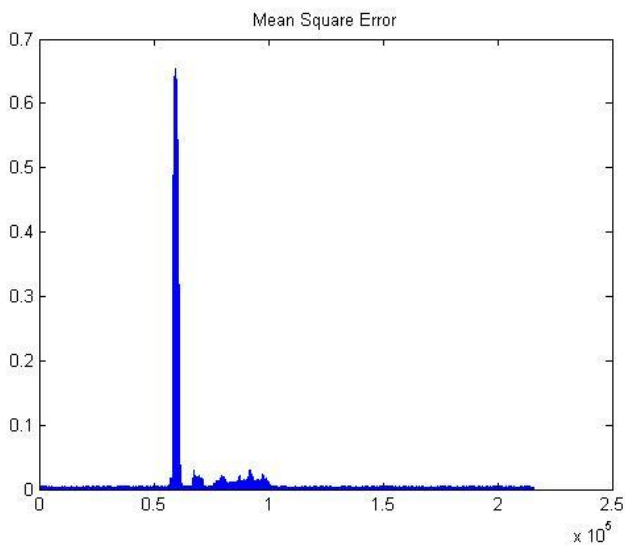

Fig.14 Mean Square between Clean and Denoised signal

A digital Chebyshev type 2 low pass filter for de-noising the speech signal is designed. In this, it is difficult to denoise the speech signal without affecting its quality. Hence the output signal from the filter has large mean square error. Because of high means square error value the SNR and PSNR values are low but the computational time is very small as compared to other filters.

\section{Kalman Filter}

The Kalman filter is a mathematical method of estimating the state of a phenomenon by minimizing the mean square error of the process. It can predict the past, current and succeeding values without a precise knowledge of the system model. The main idea of the Kalman filter is to predict the future original values from the present and previous values in a reiterative way. This method is usually used when there is uncertainty in information. When the required data cannot be retrieved directly we use Kalman filter. In some situations there is unavailability of the required data hence the available data which is close to the actual data is used as input in Kalman filter. It estimates how a signal is going to behave in future. It is ideal filter for non- stationary signals. [5] Here, Kalman filter has been used to filter out random noise from a speech signal.

The process employed in this algorithm, which is recurrent, occurs in two steps. The first step is -'the prediction' wherein the filter generates estimates of the present state variables, including the uncertainties they are associated with. The second step is called 'the estimation'. Based on the next measurement's outcome the estimates are updated with the weighted average. Higher weights are assigned to estimates with greater certainty. [6]

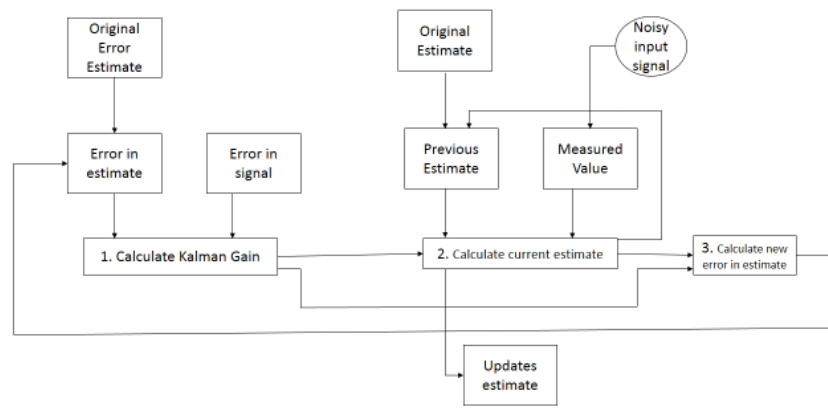

Fig.15 Kalman Filter Methodology
The results of Kalman Filter with graphs of the original, noisy, denoised signals and the MSE plot have been illustrated below.
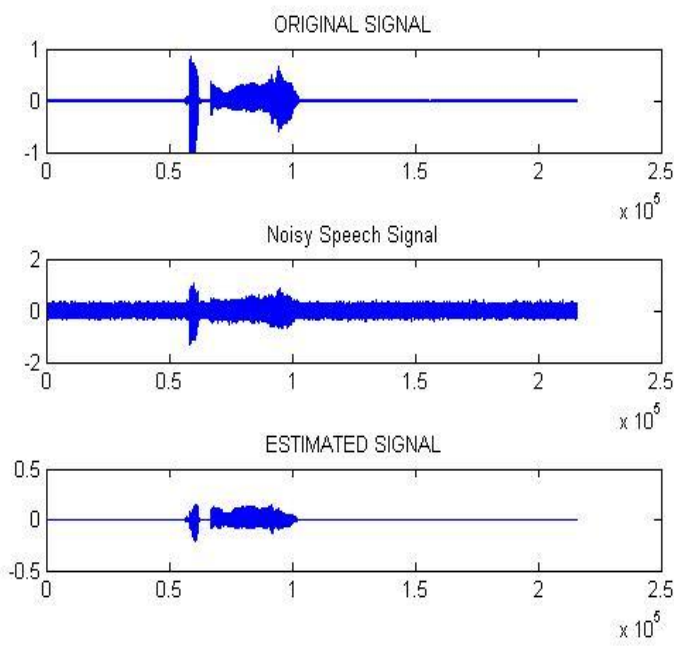

Fig.16 Original, Noisy and Estimated Signals

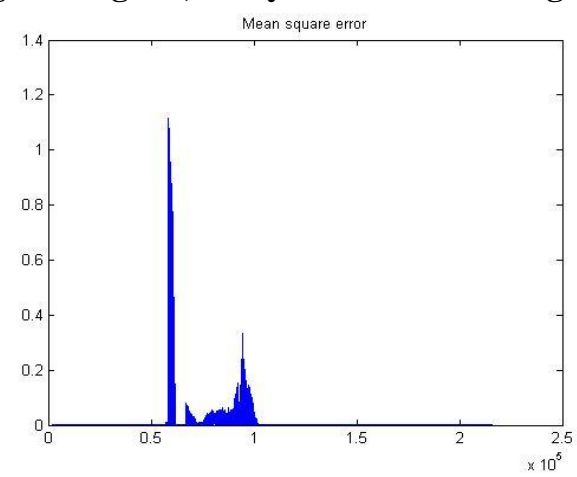

Fig.17 Error between denoised and original signal

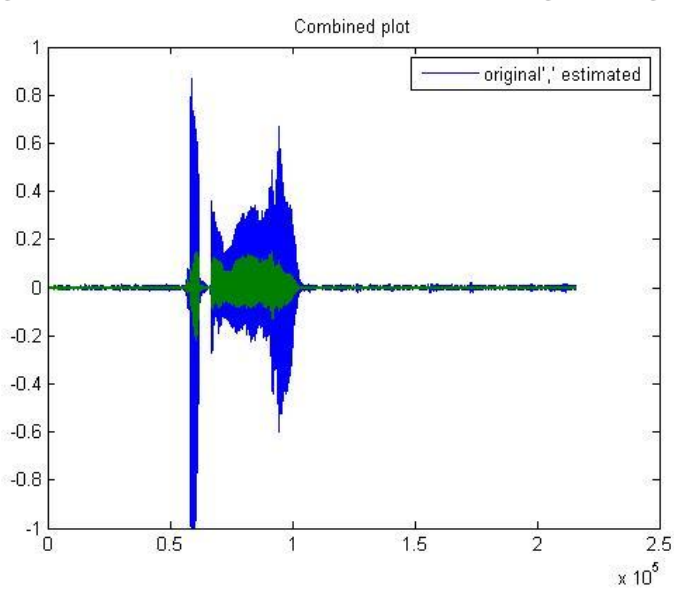

Fig 18. Combined plot of original signal and estimated signal

The Kalman Filter gives satisfactory results by filtering out noise from the original speech. The mean square error algorithm is used to find out parallelism between the original speech signal and the output of Kalman filter. The main advantage of this filter is that it is quick and has fast processing. [7] It uses only the present values and the values calculated just prior to them. It doesn't need any more past information. The memory only contains the values of

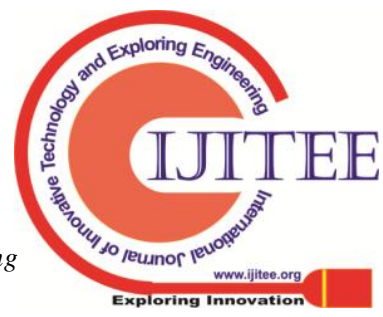




\section{A RESEARCH ON DIFFERENT FILTERING TECHNIQUES AND NEURAL NETWORKS METHODS FOR DENOISING SPEECH SIGNALS}

previous state. However, this method might not work for real time applications. This may be due to inaccuracies in modelling and errors in the assumptions made.

\section{THE NEURAL NETWORK METHOD \& RESULTS}

In artificial neural network method a basic algorithm ADALINE (Adaptive Linear Neuron) is used for noise cancellation. The second method includes de-noising the speech signals using deep learning. There are two types of deep learning networks used for de-noising speech signals. The first deep learning method is by using fully connected layers neural networks and the second method includes using convolution layers neural networks for speech signals de-noising.

Deep learning, otherwise called hierarchical learning, uses logic just like human brain to process the data. Deep learning model examines the data using ANN. ANN functions as an algorithm having layered structure to extract 'information' from the data. This information is specific features of data. This information is extracted piece by piece by each layer. Each layer brings out different features from a data set. [16] Hence DL is a hierarchical model having multiple levels of abstraction. DL networks are prior trained by past experiences. Neural networks are substructure in deep learning to process the data and extract information. Neural networks use neurons as network of connection to transfer the data as input and output values. They take many data sets as training example and build a system which can identify a feature from the training sets. They do not possess any past information about the data but develops a structure for feature identification from the data. [17]

\section{A. ADALINE}

Adaptive Linear Neuron, otherwise called ADALINE is a single layered neural network. Its structure consists of a feedback which enables one to compare the current output with the value that is desired. Once the values are compared (based on the training algorithm), updating of bias and weights takes place. It calculates the sum of products using weight vectors and input and gives a single output value. It uses the delta rule to modify the rates iteratively and hence this neural network is able to respond to any change in the environment in which it operates. An adaptive ADALINE is designed which is best suited for non-stationary signals. The aim is to modify the weights and biases in a way that it minimizes the sum of square errors between the network output and the targets. It is based on LMS algorithm. A function called 'adapt' is used in MATLAB for the same.

The performance of ADALINE neural network is largely dependent on the selection of right learning rate value. The learning rate updates the weights of the network which minimizes the error. The training speed of the neural network is also influenced by the learning rate. The learning rate value should neither be too small nor too large. The paper aims to determine the values of learning rate for which the network de-noises speech signal satisfactorily. [15] The performance of adaptive ADALINE with variation in learning rate is illustrated below. The results of ADALINE is also illustrated below.
Table.2 Variation of MSE and Execution time with variation in learning rate

\begin{tabular}{|c|c|c|}
\hline LEARNING RATE & $\begin{array}{c}\text { MEAN SQUARE } \\
\text { ERROR }\end{array}$ & $\begin{array}{c}\text { EXECUTON } \\
\text { TIME }\end{array}$ \\
\hline 0.01 & 0.0022 & $691.315 \mathrm{~s}$ \\
\hline 0.05 & 0.0011 & 535.668 \\
\hline 0.08 & $8.68 \times 10^{-4}$ & 468.442 \\
\hline 0.09 & $8.25 \times 10^{-4}$ & 530.239 \\
\hline 0.1 & $7.55 \times 10^{-4}$ & 432.388 \\
\hline 0.2 & $4.58 \times 10^{-4}$ & 450.018 \\
\hline 0.3 & $6.38 \times 10^{-4}$ & 405.501 \\
\hline 0.4 & 0.5279 & 511.479 \\
\hline
\end{tabular}

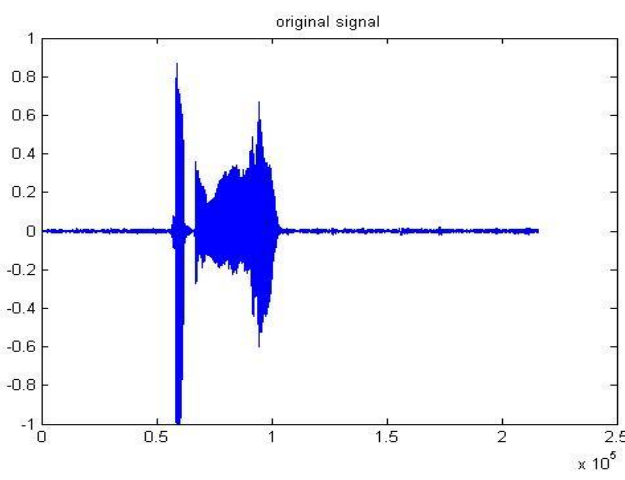

Fig.19 Clean Audio

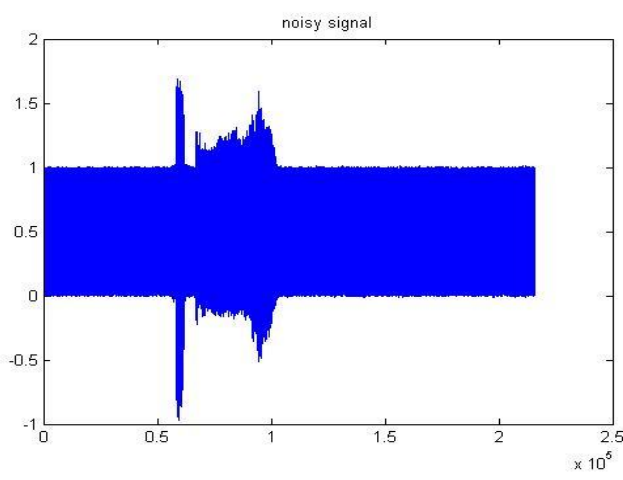

Fig.20 Audio with added Noise

filter output

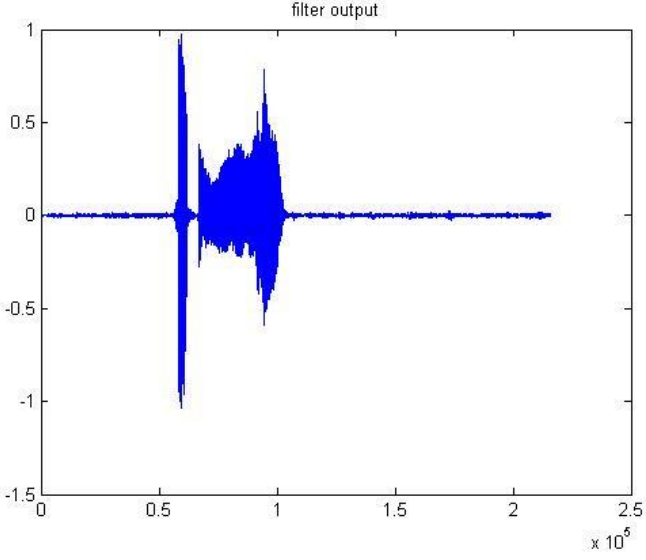

Fig.21 Denoised signal 


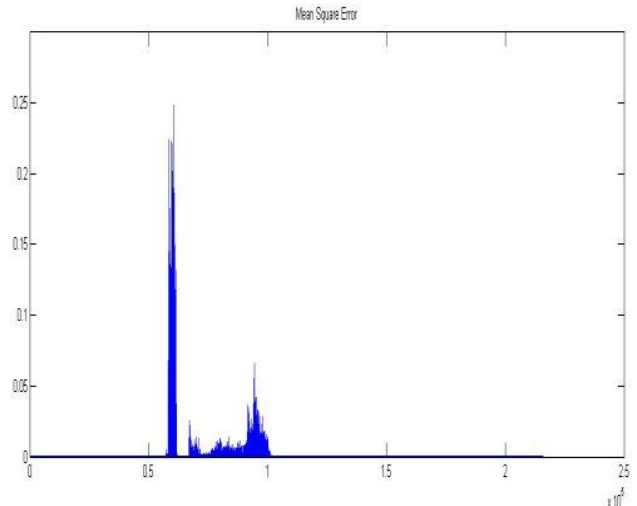

Fig.22 Mean Square Error

The Adaptive ADALINE neural network is designed in MATLAB software. ADALINE is the most basic neural network which can be incorporated for speech de-noising. Being an adaptive liner algorithm it abates the noise from the signal instead of filtering the noise from the noisy signal. The network works well and gives high PSNR and SNR values. The speech distortion is minimal and there is almost insignificant difference between filtered output and original signal. The only drawback of this method is that it is time consuming. The success of this method is highly influenced by the learning rate of the network. From the data it is clear that the learning rate should be in the range of $0.08-0.3$, as these learning rates provide low mean square error.

B. Deep Learning Fully Connected and Fully Convolutional Neural Networks.

\section{Fully Connected Network:}

The architecture of fully connected neural network contains neurons which are linked with every other neuron in the preceding layer. The connection is developed via weights.

\section{Fully Convolutional Network:}

In the architecture of convolutional neural network the neuron has connections only with few neurons from the preceding layer. The weight used in every neuron is the same. It can be said that the neurons are locally connected sharing the layers of weight. [18][19].The CNN has three major layers: the input layer, the output layer and the hidden layer. The hidden layers comprises of the Convolutional layer, Activation layer, Pooling layer, Fully Connected layer and Normalised layer. The Convolution Layer merges the data by mathematical operation. In Activation Layer the result from the Convolution layer is passed through the ReLU activation layer. In Pooling Layer the dimensionality of parameters is reduced. The training time is shortened in pooling layer. The Fully Connected Layer converts the three dimension output from pooling layer to a one dimension output and the Normalised Layer normalizes the output.

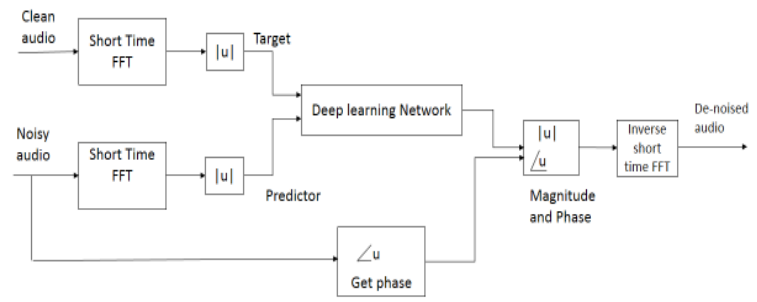

Fig.23 Deep Learning Methodology
The audio is transformed into the frequency domain by employing STFT (Short Time Fourier Transform). The window chosen is a hamming with the length being 256 samples and the overlap being $75 \%$. The spectral vector's size is reduced to 129. Eight successive noisy STFT vectors form the input of the predictor and the estimate each output is calculated using the present noisy vector and the seven previous ones. Here, the predictor is the original signal combined with noise and the target is assumed to be the original audio. To reduce the amount of computation involved, both the original and noisy audios are down sampled to 8 Kilo Hertz. The predictor is magnitude spectrum of noisy signal, the Target is the magnitude spectrum of clean signal, and the output is the magnitude spectrum of denoised signal.

A regression network is used to reduce the MSE (Mean Square Error) between output and target as much as possible. By making use of the magnitude spectrum of the output and the noisy signal's phase, the denoised speech is obtained in the time domain.

The results of the deep learning methods using Fully connected and Fully Convolutional Neural Networks are illustrated below.

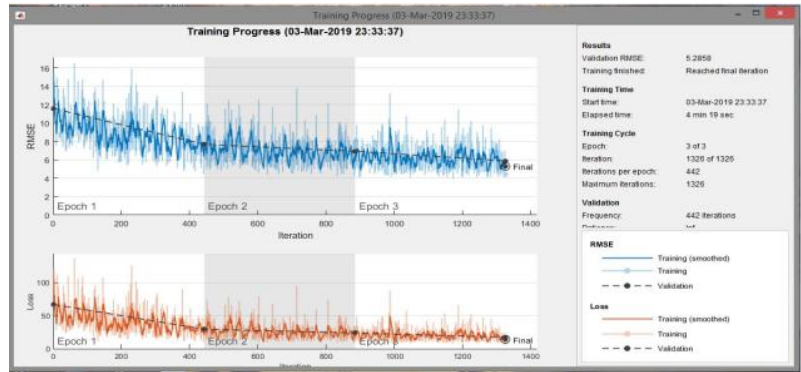

Fig.24 Training Progress
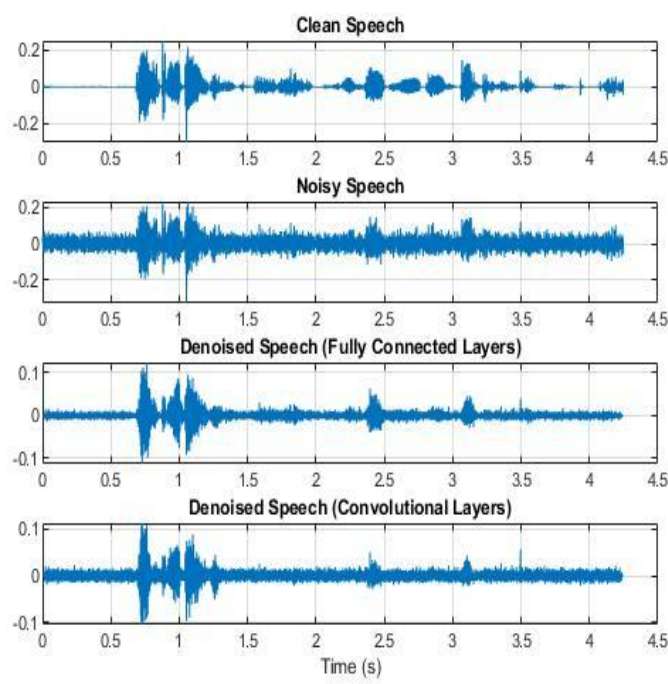

Fig.25 Clean, Noisy and Filtered signals 

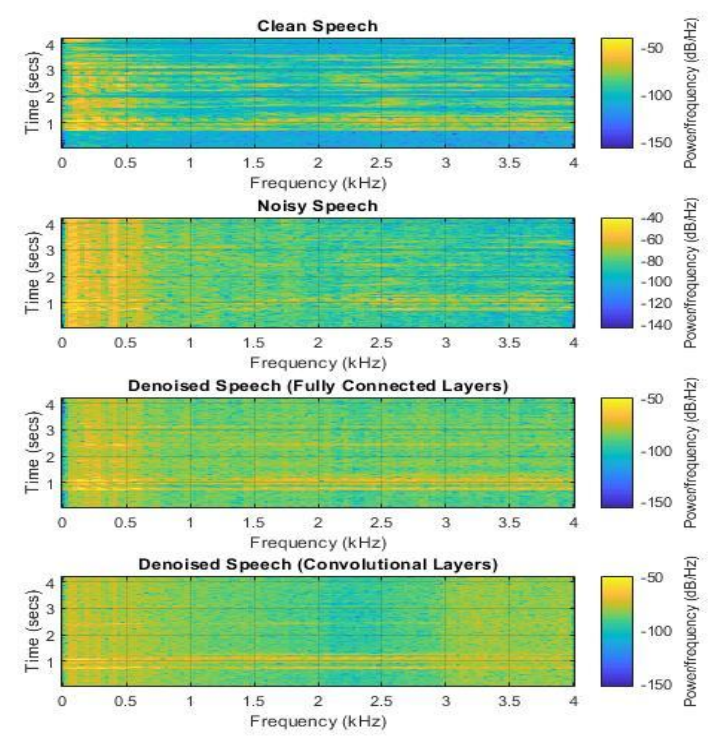

Fig.26 Spectrograms of the signals

Table .3 PSNR and SNR values for fully connected deep learning method

\begin{tabular}{|c|c|}
\hline PSNR & SNR \\
\hline 25.6543 & 4.9769 \\
\hline 32.3139 & 5.8657 \\
\hline 28.5387 & 4.6798 \\
\hline 26.4285 & 5.1652 \\
\hline 23.6139 & 4.3811 \\
\hline
\end{tabular}

Table. 4 PSNR and SNR values for fully convolutional deep learning method

\begin{tabular}{|c|c|}
\hline PSNR & SNR \\
\hline 25.2832 & 4.1441 \\
\hline 18.7651 & 3.8714 \\
\hline 26.1473 & 4.2857 \\
\hline 24.9152 & 4.8336 \\
\hline 20.5890 & 4.0621 \\
\hline
\end{tabular}

The main motive behind working with deep learning techniques is to be able to filter out real time noises such as noise of the washing machine, train etc. from the speech signal. This method has given mediocre results, the SNR and PSNR values are not very high but the de-noised signal has similarity with the original clean speech signal. However, the deep learning method has a major drawback in that it has extremely slow processing speed. Even after using parallel computing toolbox to speed up the processing it takes almost ninety minutes to de-noise a speech signal. It makes the system (laptop) slow and the battery drains out easily when this algorithm is run in MATLAB. Thus it is not ideal to use this method for practical purposes. Thus, the deep learning cannot be a practical method for de-noising speech signals at this stage.

\section{CONCLUSION}

After running 100 voice samples through each of the filters, the average PSNR and SNR values for each were calculated. They are as follows:
Table.5 Average PSNR and SNR values for all filters

\begin{tabular}{|c|c|c|c|c|c|}
\hline & $\begin{array}{c}\text { Adaptive } \\
\text { Filter }\end{array}$ & ADALINE & $\begin{array}{c}\text { Weiner } \\
\text { filter }\end{array}$ & $\begin{array}{c}\text { Chebyshev } \\
\text { Filter }\end{array}$ & $\begin{array}{c}\text { Kalman } \\
\text { Filter }\end{array}$ \\
\hline $\begin{array}{c}\text { Average } \\
\text { SNR }\end{array}$ & 13.2977 & 6.7229 & 1.0089 & 0.022431 & -0.46613 \\
\hline $\begin{array}{c}\text { Average } \\
\text { PSNR }\end{array}$ & 38.4618 & 31.8517 & 26.1309 & 25.30317 & 24.6559 \\
\hline
\end{tabular}

The paper focuses on the most fundamental step in speech enhancement which is noise cancellation. Overall, seven speech signal de-noising techniques have been used. The detailed analysis of each filtering method is done and a comparison has been drawn based on performance of each de-noising technique. The paper arrived at a conclusion that the Adaptive filter using LMS algorithm is the best suited de-noising technique for most of the speech signals. Among the neural network methods, ADALINE gives best results. ADALINE uses adaptive LMS algorithm to minimize error between network output and the targets. However, the main difference in performance of filtering and neural network de-noising methods is that the neural networks have longer execution time compared to the filtering methods. The deep learning de-noising technique is the most complex method of all. The execution time is very large in deep learning; however the results are not satisfactory. Hence, deep learning de-noising methods cannot be used for speech applications.

\section{REFERENCES}

1. Rodriguez, J. E. F. F. R. E. Y. J., Lim, J., \& Singer, E. (1987, April). Adaptive noise reduction in aircraft communication systems. In ICASSP'87. IEEE International Conference on Acoustics, Speech, and Signal Processing (Vol. 12, pp. 169-172). IEEE.

2. Kumari, M., Talukdar, N., \& Ali, I. (2016, November). A new gender detection algorithm considering the nonstationarity of speech signal. In 2016 2nd International Conference on Communication Control and Intelligent Systems (CCIS) (pp. 141-146). IEEE.

3. Liu, S., Jin, Y., Yu, H., \& Yang, L. (2015, September). Study on the acoustic characteristics of speech and physiological development of vocal organs for two-yearold children. In 2015 Fifth International Conference on Instrumentation and Measurement, Computer, Communication and Control (IMCCC) (pp. 576-579). IEEE.

4. Wang, H., Lu, Z., Zhao, H., \& Feng, H. (2015, September). Application of Parallel Computing in Robust Optimization Design Using MATLAB. In 2015 Fifth International Conference on Instrumentation and Measurement, Computer, Communication and Control (IMCCC) (pp. 1228-1231). IEEE.

5. Li, C., \& Andersen, S. V. (2004, November). Integrating Kalman filtering and multi-pulse coding for speech enhancement with a non-stationary model of the speech signal. In Conference Record of the Thirty-Eighth Asilomar Conference on Signals, Systems and Computers, 2004. (Vol. 2, pp. 2300-2304). IEEE. 
6. Fujimoto, M., \& Ariki, Y. (2000). Noisy speech recognition using noise reduction method based on Kalman filter. In 2000 IEEE International Conference on Acoustics, Speech, and Signal Processing. Proceedings (Cat. No. 00CH37100) (Vol. 3, pp. 1727-1730). IEEE

7. Xia, Y., \& Wei, Q. (2016, July). An effective Kalman filtering method for enhancing speech in the presence of colored noise. In 2016 International Conference on Audio, Language and Image Processing (ICALIP) (pp. 469-474). IEEE.

8. Kim, K., Chung, Y., Park, C., Son, Y., \& Yoon, J. (2007, December). Speech quality enhancement based on sinusoidal model using chebyshev filter. In Future generation communication and networking (fgen 2007) (Vol. 1, pp. 323-327). IEEE.

9. Shahid, M. B., Abbasi, M. A., \& Muazzam, M. (2015, December). Detection of noise in high pass IIR digital filters. In 2015 International Conference on Humanoid, Nanotechnology, Information Technology, Communication and Control, Environment and Management (HNICEM) (pp. 1-5). IEEE.

10. Mendiratta, A., \& Jha, D. (2014, January). Adaptive noise cancelling for audio signals using least mean square algorithm. In International Conference on Electronics, Communication and Instrumentation (ICECI) (pp. 1-4). IEEE.

11. Gupta, P., Patidar, M., \& Nema, P. (2015, September). Performance analysis of speech enhancement using LMS, NLMS and UNANR algorithms. In 2015 International Conference on Computer, Communication and Control (IC4)(pp. 1-5). IEEE.

12. Jingfang, W. (2011, August). Noisy speech in real time iterative Wiener filter. In 2011 International Conference on Mechatronic Science, Electric Engineering and Computer (MEC) (pp. 2102-2105). IEEE.

13. Plapous, C., Marro, C., \& Scalart, P. (2006). Improved signal-to-noise ratio estimation for speech enhancement. IEEE Transactions on Audio, Speech, and Language Processing, 14(6), 2098-2108.

14. Fah, L. B., Hussain, A., \& Samad, S. A. (2000). Speech enhancement by noise cancellation using neural network. In 2000 TENCON Proceedings. Intelligent Systems and Technologies for the New Millennium (Cat. No. 00CH37119) (Vol. 1, pp. 39-42). IEEE

15. Darken, C., Chang, J., \& Moody, J. (1992, August). Learning rate schedules for faster stochastic gradient search. In Neural Networks for Signal Processing II Proceedings of the 1992 IEEE Workshop (pp. 3-12). IEEE.

16. Liu, D., Smaragdis, P., \& Kim, M. (2014). Experiments on deep learning for speech denoising. In Fifteenth Annual Conference of the International Speech Communication Association.

17. Alom, M. Z., Taha, T. M., Yakopcic, C., Westberg, S., Sidike, P., Nasrin, M. S., ... \& Asari, V. K. (2019). A State-of-the-Art Survey on Deep Learning Theory and Architectures. Electronics, 8(3), 292.

18. Erseven, M., \& Bolat, B. (2018, May). Regression-based speech enhancement by convolutional neural network. In 2018 26th Signal Processing and Communications Applications Conference (SIU) (pp. 1-4). IEEE.

19. Park, S. R., \& Lee, J. (2016). A fully convolutional neural network for speech enhancement. arXiv preprint arXiv:1609.07132.

20. Gopichand, G., \& Saravanaguru, R. A. K. (2016). A Generic Review on Effective Intrusion Detection in Ad hoc Networks. International Journal of Electrical \& Computer Engineering (2088-8708), 6(4).

21. G. Gopichand, R.A.K. Saravanaguru, K. Ramesh Babu, Fully secured intrusion detection system for sensing attacks in MANET, Journal of Advanced Research in
Dynamical and Control Systems, vol. 10, no. 4 Special Issue, pp. 810-816, 2018

22. Gopichand G, Saravanaguru RA.K., Collaborative Packet Dropping Intrusion Detection in MANETs, Recent Patents on Computer Science (2019) 12: 1. https://doi.org/10.2174/2213275912666190618163426

23. Gopichand G., Sankeerth K.S., Parlapalli A, Evaluation of recommendation systems using trust aware metrics, International Journal of Recent Technology and Engineering, Volume-7, Issue-6S4, April 2019

24. Gopichand G, Vishal Lella, Sai Manikanta Avula, Enhancing Performance of Map Reduce Workflow through H2HADOOP: CJBT, International Journal of Recent Technology and Engineering, Volume-7, Issue6S4, April 2019

25. Gopichand G, Sailaja G, N. VenkataVinod Kumar, T. Samatha, Digital Signature Verification Using Artificial Neural Networks, International Journal of Recent Technology and Engineering, Volume-7 Issue-5S2, January 2019

26. Gopichand G, Ra.K.Saravanaguru, .K.Ramesh Babu, Usage of AODV and AOMDV Protocols in Perceiving Black hole Attacks in a MANET, International Journal of Pharmacy \& Technology,Volume 8, Issue 4,December 2016

27. Mehta M., Rajesh Mamilla, Sunithavenugopal, Gopichand G, Growth and development of start-ups in India - A study with respect to mechanical and production engineering, International Journal of Mechanical and Production Engineering Research and Development, Volume : 8-2, April 2019

28. Jitesh Shaw, P. M. Durai Raj Vincent, Senthilnathan Palaniappan, *, Arun Kumar Sangaiah, Gopichand G, Intelligent Phishing Detection System Using Feature Analysis, Journal of Computational and Theoretical Nanoscience Vol. 15, 2533-2538, 2018

29. Senthilnathan Palaniappan,Saiprasad Palli, Gopichand G, Sirajudeen Ameerjohn, Siva Shanmugam Gopal, Enhanced Handwritten Number Detection Using Kernel Discriminant Analysis (KDA), Journal of Computational and Theoretical Nanoscience Vol. 15, 2539-2543, 2018

30. H R Swathi, Shah Sohini, Surbhi, Gopichand G, Image compression using singular value decomposition, IOP Conference Series: Materials Science and Engineering 263(4).

31. Santhi H, Gopichand G, Gayathri P, Automated Smart Parking System using IoT, Journal of Advanced Research in Dynamical \& Control Systems, Vol. 10, 09Special Issue, 2018

32. P Gayathri, Mayank Agarwal, H Santhi, Gopichand G, Bone Breakage Identification Using Image Processing Techniques, Journal of Advanced Research in Dynamical \& Control Systems, Vol. 10, 09-Special Issue, 2018 